DOI: https://doi.org/10.14232/actahisp.2020.25.23-35

\title{
COLOMBIANAS EN CHILE: TENDENCIAS Y DETERMINANTES DE LA MIGRACIÓN FEMENINA
}

\author{
Amadea BATA-BALOG - GABRIElla Thomázy
}

Universidad Nacional de Servicio Público, Hungría

\begin{abstract}
Resumen: La migración internacional Sur-Sur ha crecido significativamente en la última década y Chile es uno de los países receptores de la migración regional. Es así, que el número de los colombianos se multiplicó en los últimos 10 años, destacando en la migración colombiana el aspecto femenino, ya que muchas mujeres buscan un empleo remunerado en el país andino, dejando a sus hijos y familiares en el país de origen. El presente estudio tiene como objetivo principal constatar cuáles son las tendencias actuales y los nuevos desafíos de las colombianas en Chile: las estadísticas disponibles, entrevistas y otros datos permiten analizar las tendencias. ¿Cuáles son los desafíos más importantes de las colombianas? ¿Por qué vienen a Chile? ¿Y cómo ha cambiado la vida de ellas con las recientes dificultades que impuso la llegada de la covid-19?

Palabras Claves: migración femenina, colombianas, Colombia, Chile.
\end{abstract}

Abstract: International South-South migration has grown significantly in the last decade; Chile emerges to be one of the most popular recipient countries of regional migration. The number of Colombians multiplied in the last 10 years, and the matter of the fact is that Colombian migration has a feminine facet, as many women seek paid employment in the Andean country, leaving their children and relatives in the country of origin. The main objective of this study is to verify the current trends and new challenges of Colombian women in Chile. Available statistics, interviews and other data allow the analysis of current trends. What are the most important challenges for Colombian women? Why do they come to Chile? And how have their lives changed with the recent difficulties imposed by the arrival of Covid-19?

Keywords: Female Migration, Colombians, Colombia, Chile.

\section{Introducción}

Durante las últimas décadas, Chile se ha convertido en un destino privilegiado de los flujos migratorios internacionales de países latinoamericanos. La migración internacional Sur-Sur ha crecido desde la crisis económica de 2008 e inició la búsqueda de otros destinos, como por ejemplo, Chile. Colombia tiene proyecciones de crecimiento a largo plazo, pero todavía registra el mayor número de adultos en la región que planifican vivir en el extranjero (IOM, 2017). Los colombianos anteriormente preferían viajar a los EE.UU., Venezuela o hacia Europa, y en especial a España, pero la tendencia cambió como consecuencia de la crisis financiera y económica de 2008, además de las barreras migratorias hacia los EE.UU., teniendo como resultado, que la migración intrarregional se ha convertido en una opción para muchos colombianos (Datos migratorios, 2020). 
Colombianas en Chile: tendencias y determinantes de la migración femenina

El presente estudio tiene como objetivo principal constatar cuáles son las tendencias actuales y los nuevos desafíos de las colombianas en Chile. Este estudio se realizó recopilando datos, estadísticas disponibles, principalmente del DEM (Departamento de Extranjería y Migración) y del INE (Instituto Nacional de Estadísticas), además, entrevistas personales permitieron analizar las tendencias de los últimos años y las principales dificultades con la llegada de la covid-19. El estudio, inicialmente, busca encontrar convergencias y divergencias entre Chile y Colombia, especialmente, en los aspectos que favorecen la migración; en el capítulo siguiente se pone énfasis en las características de la migración internacional en Chile, centrándose en los colombianos, y finalmente se identifican los patrones de movilidad ocupacional, en concreto, de la migración femenina colombiana.

\section{Chile vs. Colombia}

Chile es un país atractivo para los migrantes colombianos, algunos de los datos económicos más importantes, como el PIB anual: 282,318 miles de millones de dólares, el PIB per cápita: 15.010 dólares, la tasa de pobreza: 8,6\%, el crecimiento del PIB para el 2021 se estima en un 3,1\% anual y la esperanza de vida es de 80 años, siendo estos indicadores los mejores en la región después de Uruguay con una población de 18.952.038 habitantes (World Bank, 2019a). En Colombia, aunque sus indicadores económicos están mejorando en los últimos años, los datos son más bajos que los chilenos, principalmente, la tasa de pobreza: 27\%, el PIB per cápita: 6.510 dólares, menos de la mitad que el chileno, con una población en crecimiento que ya superó los 50 millones en el 2019. La esperanza de vida aumentó considerablemente, alcanzando los 77 años en 2019, mientras en 2005 apenas llegó a los 74 años (World Bank, 2019b).

La mayor diferencia entre los dos países se puede ver en el Índice de Percepción de la Corrupción, elaborado por la ONG Transparencia Internacional (2019). En este índice Colombia obtuvo el lugar n ${ }^{\circ} 96$ entre 198 países, mientras que Chile, permanece como el segundo país menos corrupto a nivel latinoamericano, ocupando en el ranking mundial la posición n ${ }^{\circ} 26$ (a modo de ejemplo, Francia ocupa el lugar n²1). Colombia está considerada como un país menos seguro que Chile, a pesar del proceso de paz y lucha contra el narcotráfico. En Colombia, la tasa de homicidios es de 25,4 homicidios por cada 100.000 habitantes, similar a México y Guatemala, mientras que la tasa de homicidios de Chile es 2,6 homicidios por cada 100.000 habitantes, convirtiéndolo una vez más en el país más seguro de América Latina en el 2019 (Insight Crime, 2019). Notable es la diferencia en el índice de desarrollo humano (HDI) en el caso de Colombia $\mathrm{n}^{\circ} 79$, mientras que Chile está en el puesto $\mathrm{n}^{\circ} 42$ en el ranking, (a modo de ejemplo, Hungría no 43 y Portugal no 40) (HDI, 2019).

Un factor muy importante para los migrantes que buscan un nuevo futuro es el sueldo. Los ingresos chilenos son de los más altos en América del Sur, principalmente, en los puestos que requieren educación terciaria (Thomázy, 2020: 412), ya que es 
bastante atractivo para los migrantes, pero trabajando en el comercio o la construcción, también se pueden conseguir ingresos elevados. Durante los últimos años, miles de residentes del suroeste de Colombia, en especial de las regiones del Pacífico y del Valle del Cauca, afectadas por el aumento de la siembra de coca, viajaron a Chile para buscar empleo en las ciudades con gran oferta de trabajo como, por ejemplo, Antofagasta (norte de Chile), que tiene un ingreso por persona de US\$37.000 anual, una cifra tan alta como en algunos países europeos (Pardo, 2018).

Chile llegó a ser el tercer destino más popular entre los colombianos (ONU, 2019), lo cual, se refleja en las remesas de dinero ${ }^{1}$ hacia Colombia. De hecho, hasta hace cinco años, Chile no aparecía entre los tres primeros orígenes de dinero a nivel mundial, pero en 2019 llegó al tercer puesto, después de EE.UU. y España, solo en ese año se enviaron desde Chile más de US\$ 400 millones (López, 2020; Banco de la República, 2019). Según las estimaciones del Ministerio de Relaciones Exteriores de Colombia, cerca de 4,7 millones de colombianos viven en el exterior, es decir, cerca del 10\% de la población vive fuera de su país, además la migración tiene un aspecto femenino, de ese total, el 54,41\% corresponde a mujeres (DNP, 2017; ONU, 2019).

A través de los perfiles de Colombia y Chile, se pueden observar varias diferencias, básicamente en los determinantes políticos (estabilidad, nivel de corrupción), económicos (PIB per cápita, ingresos, oportunidades de trabajo), sociales (pobreza, HDI, seguridad), -actuando como factores de push y pull- y finalmente los determinantes culturales (idioma) y espaciales (cercanía de Chile) - actuando como factores de pull en las decisiones migratorias. Los indicadores macroeconómicos favorecen a los colombianos que eligen la migración, es decir, que en muchos aspectos Chile es considerablemente más atractivo para los colombianos y una opción racional del destino (Bata-Balog - Thomázy, 2020).

\section{Caracterización general de los inmigrantes colombianos en Chile}

Chile ha experimentado un fenómeno migratorio muy significativo en la última década, según una estimación realizada en conjunto por el INE y el DEM en 2002, 195.320 extranjeros vivían en Chile, mientras que el número de personas extranjeras residentes habituales en Chile alcanzó 1.492 .522 el 31 de diciembre del 2019, de las cuales 763.776 correspondieron a hombres y 728.746 a mujeres (DEM, 2019).

La gran mayoría de los inmigrantes internacionales que llegan a Chile son de origen latinoamericano, siendo actualmente Colombia el cuarto país desde donde provienen la mayoría de estos migrantes. Según las estimaciones, 161.153 colombianos vivían en Chile en 2019, un 10,8\% del total de la población extranjera residente en Chile (DEM, 2019), mientras que en 2015 los colombianos ocuparon el segundo lugar, después de los

${ }^{1}$ Remesas: ayuda financiera a los familiares en su país de origen, mientras se prepara el regreso de los donantes manteniendo y fortaleciendo su capital económico y social (Albertini et al., 2019: 1700). 
Colombianas en Chile: tendencias y determinantes de la migración femenina

peruanos, representando el 13,6\% de los extranjeros en Chile (Gissi et al, 2019: 132). En los últimos dos años con la llegada masiva de los haitianos y venezolanos, estos ya superaron a los colombianos.

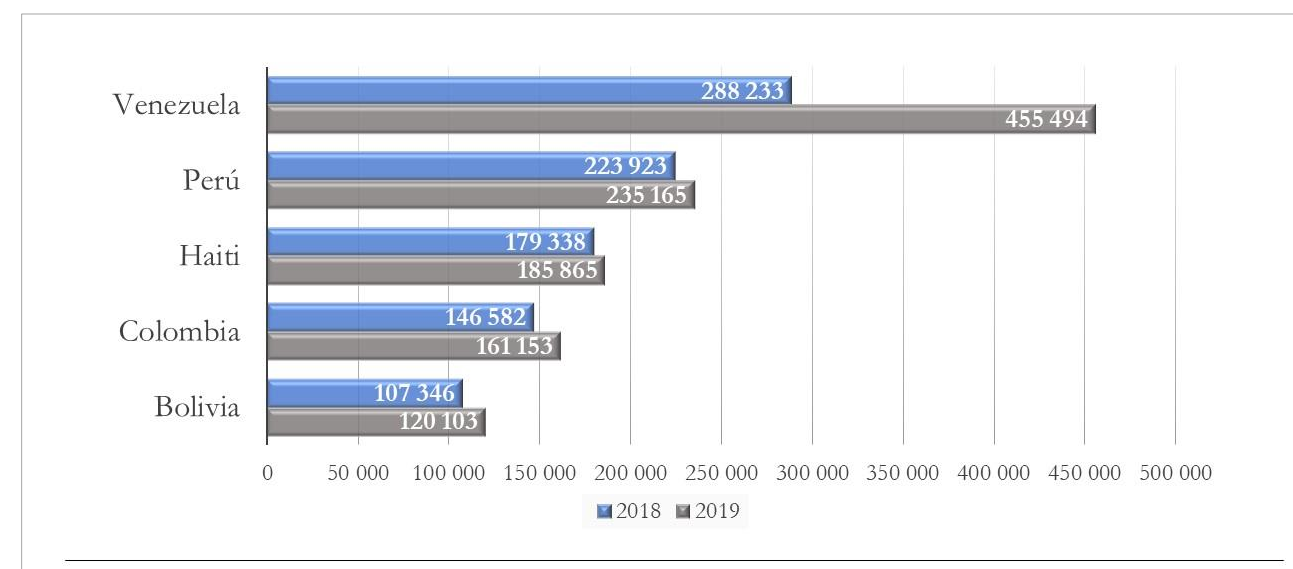

Gráfico 1. Número de personas estimadas según país de nacimiento (2018-2019)

Fuente: adaptado de Autor por datos de INE-DEM

La ley de migración en Chile es la más antigua de Sudamérica y facilita la llegada de los inmigrantes, ofreciendo diferentes visas para los extranjeros que quieran vivir en el país. Generalmente los extranjeros ingresan al país con visa turista y solicitan una "visa temporaria" en Chile, en caso de que los colombianos requieran un certificado de antecedentes también. Muchos de los inmigrantes se quedan ilegalmente en el país después del vencimiento de la visa de turista o llegan a Chile desde pasos fronterizos no habilitados. Actualmente una nueva ley migratoria avanza en el Congreso y busca modernizar la actual regulación, obligando en el futuro a solicitar la visa solamente en el país de origen ("Polémica...", 2020). Según los estudios relacionados con los colombianos, ellos generalmente viajan a Chile con la ayuda de familiares, parientes o amigos que ya viven en el país (Fernández et al, 2020; Gissi et al, 2019), así el cambio de la ley no afectaría tanto a la inmigración colombiana, pero sería más difícil legalizar el estado de las personas que ya viven en Chile de forma ilegal.

Se puede establecer que ciertas características de la migración generalmente se concentran en la Región Metropolitana de Santiago (Baeza, 2019: 3). Además, entre los colombianos es bastante popular Antofagasta, la ciudad minera más importante del país, llamada por muchos como "Antofalombia" por la cantidad de colombianos que viven en ella. Según las cifras oficiales de 2018 se estima que una cuarta parte del total de los colombianos vive en Antofagasta, además que el $16 \%$ de la población son 
extranjeros (Prado, 2018). Mientras que en Chile hay grupos de migrantes que se consideran de estratos socioeconómicos altos, como los europeos, de estratos medios, generalmente los brasileños, y de estratos bajos, como los bolivianos y haitianos, la diferenciación socioeconómica en caso de los colombianos es muy diversa (Gissi Ghio, 2017: 7).

La población inmigrante, mayor de 25 años, nacida en Colombia posee una escolaridad promedio de 12,1 años, siendo la cifra de 12,0 para hombres y ligeramente mayor para las mujeres con 12,2, es decir, que esta población, en promedio, tiene completa la educación secundaria (INE, 2018: 47). Los extranjeros poseen en promedio 12,6 años de escolaridad en Chile: los peruanos 11,6, los bolivianos 10,6 y los venezolanos superan a todos los grupos con 15,6 años de escolaridad, a saber, la mayoría de los venezolanos tienen educación terciaria. Como contraparte, las personas nacidas en Chile tienen una escolaridad promedio de 11 años, es decir, los inmigrantes tendrían una mayor cantidad de años de escolaridad (INE, 2018: 87). Colombia tiene un acuerdo bilateral para convalidar los títulos profesionales en Chile, pero con la convalidación no se permite trabajar libremente, además, si la profesión no existe en Chile, tienen que solicitar un costoso trámite en la Universidad de Chile (Thomázy, 2020).

Como los colombianos generalmente no tienen educación terciaria, normalmente trabajan en diferentes sectores, como por ejemplo, el comercio, la hotelería, las actividades domésticas y la construcción (Contreras, 2018). Los colombianos son famosos por los "Barbershop" y muchos trabajan como camareros o taxistas, siendo considerados más serviciales que los chilenos, por eso se les prefiere en el momento de contratar el sector servicios.

\begin{tabular}{|l|c|}
\hline Sector & Porcentaje \\
\hline Comercio & $23,8 \%$ \\
\hline Actividades de los hogares & $18,2 \%$ \\
\hline Manufactura & $14,9 \%$ \\
\hline Alojamieno y serivicios de comida & $10,2 \%$ \\
\hline Construcción & $5,7 \%$ \\
\hline Salud & $4,6 \%$ \\
\hline Otro & $22,5 \%$ \\
\hline \multicolumn{2}{|c|}{ Gráfico 2. ¿Dónde trabajan los colombianos? / Por sectores económicos } \\
\hline
\end{tabular}


Colombianas en Chile: tendencias y determinantes de la migración femenina

La proporción entre los trabajadores chilenos y extranjeros que pueden trabajar en una misma empresa está limitada en Chile, la legislación laboral establece que: "[...]el $85 \%$ a lo menos, de los trabajadores que sirvan a un mismo empleador debe ser de nacionalidad chilena [...]". Sin embargo, se exceptúa de esta disposición al empleador que no contrata a más de veinticinco trabajadores, considerándose como chileno al extranjero cuyo cónyuge, sus hijos sean chilenos o residentes por más de cinco años en el país. Así puede contratarse a colombianos con mayor facilidad en pequeñas empresas, ya que si estas poseen menos de 25 trabajadores, la ley antes mencionada no sería aplicable (Ley No , 18.620, 2003; Thomázy, 2020: 413).

La integración de los colombianos no ha sido fácil, frecuentemente se describe a la sociedad chilena como el opuesto de la colombiana, especialmente en cuanto a su calidez, ya que los chilenos son fríos y poco amigables. La integración incluye, por ejemplo, el dominio completo de los modismos chilenos (Gissi et al, 2019: 133). Es importante mencionar que la sociedad chilena está llena de estereotipos, asociándose frecuentemente a los colombianos con el narcotráfico, la delincuencia y la prostitución (Fernández et al, 2020; Gissi - Ghio, 2017).

\section{Migración Femenina - las colombianas en Chile²}

Según la estimación de la Organización Internacional del Trabajo (OIT), 164 millones de personas son trabajadores migrantes en el mundo, entre ellos hay menos mujeres que hombres. Según las estimaciones, el 58,4\% son hombres y el 41,6\% son mujeres, mientras que en América Latina, una de las principales características de la migración es el aspecto femenino, más de la mitad (el 51,4\%) de los migrantes internacionales son mujeres. Esto significa que muchas mujeres viajan por cuenta propia y buscan trabajo sin ser una carga para su pareja, marido u otro miembro familiar. Las mujeres migrantes tienen desventajas laborales en relación con los hombres migrantes y con las mujeres locales, además sufren otro tipo de discriminaciones: origen étnico, mujer extranjera, situación de vulnerabilidad económica, etc. Las mujeres migrantes corren un mayor riesgo de explotación y abuso, además aquellas que son cualificadas tienen menor posibilidad de encontrar trabajos afines $\mathrm{y}$, las no cualificadas, trabajan en sectores ilegales o menos visibles que los hombres migrantes (ILO, 2018: IX; ILO, 2017: 2-4; O’Neil et al., 2016).

Muchas de las mujeres migrantes viajan sin familia mientras trabajan en otro país, dejan a sus hijos en el país de origen al cuidado de otras mujeres o parientes, así desarrollando "cadenas globales de cuidados"3. A partir de 2001, muchas colombianas

\footnotetext{
2 Para este estudio se entrevistaron a 5 personas locales que contrataron a colombianas por diferentes motivos, además uno de los voluntarios del Centro Abierto de la Reina, un organismo sin fines de lucro que trabaja mayormente con migrantes, entre ellos muchas colombianas. Los entrevistados son de diferentes niveles socioeconómicos de Santiago.

${ }^{3} \mathrm{El}$ concepto fue establecido por Arlie R. Hochschild (Universidad de California, Berkeley).
} 
buscaron trabajo en España, principalmente como empleadas domésticas, pero la llegada de la crisis financiera y económica de 2008 inició la migración sur-sur y en este contexto, Chile se ha convertido en uno de los destinos preferidos de las mujeres dentro de la región. El trabajo de empleada doméstica se denomina oficialmente en Chile "trabajadora de casa particular" (TCP). Es importante destacar que este trabajo se convirtió en una entrada al mercado laboral en Chile, al igual que en España (Oso Martínez, 2008; Hochschild, 2001).

Como mencionamos anteriormente, una de las características importantes de los flujos migratorios hacia Chile es la tendencia a su feminización: las colombianas representan más de la mitad (53\%) de los residentes colombianos en el país en 2018 y 2019 (ILO, 2017: 6; DEM, 2018-2019). Según los datos de la INE, 77.775 colombianas vivían en Chile en 2018 y 85.051 en 2019, en ambos años el 44\% de ellas tiene una edad comprendida entre 25 y 39 años y solo un 13\% de ellas tenía 19 años o menos en 2019, mientras que en 2018 la cifra fue levemente mayor, un 14\%. La mayoría de las colombianas viven en Santiago, el segundo lugar más popular para ellas es Antofagasta, y pocas colombianas viven en el sur de Chile debido a las condiciones climáticas y a una menor oferta laboral (INE, 2019).

\begin{tabular}{|l|r|r|}
\hline \multicolumn{1}{|l|}{} & $\mathbf{2 0 1 8}$ & $\mathbf{2 0 1 9}$ \\
\hline Total País & $\mathbf{7 7 7 7 5}$ & $\mathbf{8 5 0 5 1}$ \\
\hline $\begin{array}{l}\text { Metropolitana de } \\
\text { Santiago }\end{array}$ & 44553 & 48215 \\
\hline Antofagasta & 14139 & 15219 \\
\hline Valparaíso & 3647 & 4109 \\
\hline Coquimbo & 2613 & 2762 \\
\hline Tarapacá & 2543 & 2753 \\
\hline
\end{tabular}

En términos de participación laboral, las mujeres colombianas se ocupan mayoritariamente de actividades de atención a clientes (hotelería, restaurantes, comercio) y en servicio doméstico como TCP (Fernández at al., 2020: 21). El 48,5\% de las mujeres en Chile trabaja ("48,5 \% de las...”, 2018), así que el contratar a una TCP ya no es un símbolo de estatus, sino que muchas veces es necesario y habitual debido a las extensas jornadas laborales (jornada completa: 45 horas semanales más una hora de almuerzo, Ley $\mathrm{N}^{\mathrm{o}} 20.786$, 2014), no solamente para el cuidado de los hijos, sino para 
Colombianas en Chile: tendencias y determinantes de la migración femenina

atender las necesidades de los adultos mayores, debido a que la sociedad chilena está envejeciendo y pagar un hogar de ancianos es muy costoso en Chile.

En 2015 entró en vigor la nueva ley de las TCP que reforzó sus derechos laborales, la cual tenía como objetivo mejorar las condiciones laborales (Ley No 20.786, 2014). Se estima que más del $50 \%$ de las trabajadoras de casa particular, aún trabajan sin contrato a pesar de que hace más de 5 años de que la ley entrara en vigor. Muchas veces falta conocimiento sobre la regulación de la contratación por parte del empleador y la TCP tampoco, además existe una tendencia en evitar el pago de las imposiciones (Alonso Sánchez, 2019). Actualmente, no hay datos exactos o estadísticas disponibles de las TCP extranjeras en Chile debido a los altos niveles de informalidad. Desde 2005, están disponibles los datos de las visas entregadas con motivo de esta actividad en la página del Departamento de Extranjería y Migración. De esta forma, recopilando la información de las visas de trabajo como TCP entre 2005-2019, se obtuvieron 202.160 visas de trabajo. En el gráfico $n^{\circ} 4$ se pueden observar las visas de trabajo como TCP, siendo las colombianas el tercer grupo mayor después de las venezolanas y peruanas en los últimos tres años. Cabe mencionar que las profesionales podrían haber solicitado una visa con otros fines (por ejemplo, visa temporaria para profesionales), por lo que el número de las TCP podría ser mayor.

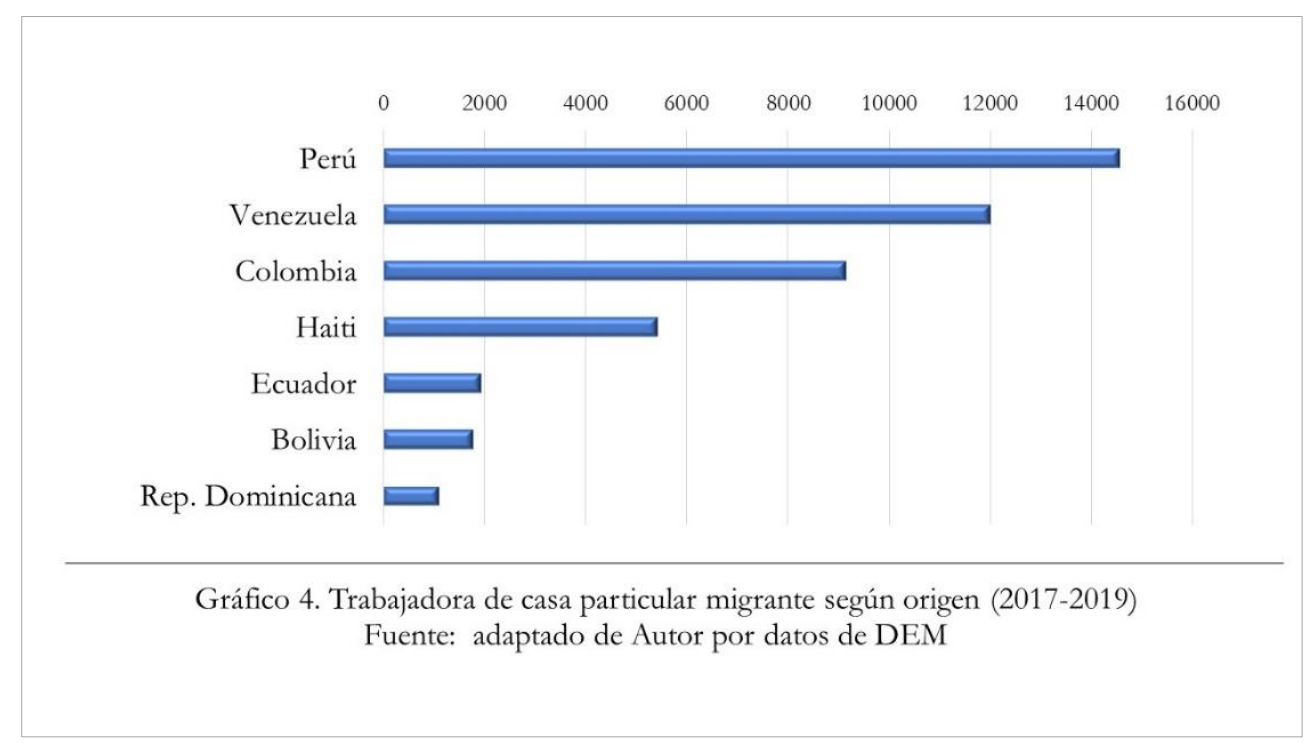

En esta área, hay un nicho laboral de las TCP de "puertas adentro" (concepto en el cual, la TCP vive con el empleador), dado que entre las chilenas esta modalidad se ha reducido de manera notable en los últimos 20 años (Arriagada - Moreno, 2011). Las chilenas prefieren trabajar de "puertas afuera" (sin vivir con el empleador), además del 
pago de sus servicios por día trabajado. Varias páginas web ofrecen servicios de búsqueda de TCP y generalmente el único filtro es la nacionalidad, pueden ganar entre US\$ 600-700 líquido 4 , alojamiento incluido, o por día US\$38-45. Uno de los entrevistados eligió una colombiana para cuidar a sus padres enfermos, pero durante la búsqueda tenía dificultad para encontrar una TCP que estuviera dispuesta a trabajar con un contrato de trabajo, debido a que muchas de las TCP quieren evitar los pagos de imposiciones y así obtener un mejor sueldo o debido a que residen ilegalmente en el país. Estas mujeres al no tener un contrato vigente son más vulnerables: “[...] ella necesita contar con un contrato [...] eso le permite estar en el país mientras recibe la visa definitiva y para nosotros fue una garantía de que trabajaría con responsabilidad pues depende de este trabajo y que no nos dejaría [...]". El caso descrito corresponde a un ejemplo en que, a veces, es una necesidad contratar a una TCP. La entrevistada, de nacionalidad chilena, trabaja durante la semana y reemplaza a su propia TCP durante el fin de semana cuidando a sus padres. Otro factor importante es que en Chile el componente afro era muy bajo en la sociedad, una variable que actualmente puede ser un motivo de rechazo: “[...] ella (la TCP de la entrevistada) es de tez blanca [...] si hubieron otras que fueron a casa de color y a mi mama no le gustan [...] la ponen nerviosa [...]".

Además de los trabajos domésticos, se observa que existe una segmentación del mercado de trabajo entre las colombianas, es decir, acceden a trabajos, sobre todo en el sector servicios, donde la informalidad también está presente, a modo de ejemplo, frecuentemente trabajan como camareras; en esta área la contratación es por un sueldo base más la propina, que es la mayor parte del sueldo, lo cual dependerá de la ubicación del local y popularidad entre turistas, pero podrían llegar a ganar cerca de US\$15002000 líquido. Cabe destacar que el sueldo promedio en Colombia es unos US\$300, las personas con menor escolaridad reciben US\$ 150 y con educación terciaria pueden ganar alrededor de US\$ 480 (Leal, 2020).

En el caso de las mujeres colombianas, la población chilena asocia su migración con la prostitución (Fernández et al, 2020), debido a las noticias del comercio sexual, frecuentemente asociadas a un fenómeno migratorio. Principalmente las mujeres afrocolombianas participan en este mercado en las ciudades mineras del norte de Chile (Pavez, 2016). Cabe mencionar que las colombianas tienen mayor vulnerabilidad para caer en grupos de trata de personas, de hecho en 2019, se desarticuló la mayor red de tráfico sexual en Chile, saliendo a la luz que algunas colombianas habrían viajado a Chile para trabajos domésticos y modelos, siendo finalmente engañadas ("El sueño de...", 2019).

Si una colombiana se quiere radicar en Chile tiene que conseguir la permanencia definitiva, la cual le permite radicar y trabajar libremente en el país, pero para eso las autoridades requieren la acreditación de sustento económico a través de un contrato de trabajo y debido a la informalidad, a veces es imposible demostrarlo. La residencia

\footnotetext{
${ }^{4}$ Precio de referencia: 740 CLP /1 USD el 09 de 2019.
} 
Colombianas en Chile: tendencias y determinantes de la migración femenina

puede obtenerse a través del matrimonio o la procreación de un hijo chileno (nacido en Chile), siendo ambas opciones denunciadas por los medios de comunicación, lo que produce la alarma xenofóbica (Pavez, 2016: 28; Thomázy, 2020).

La llegada de la covid-19 afectó a las colombianas y al igual que otros migrantes, muchas de ellas perdieron su fuente de ingresos o redujeron sus sueldos manteniendo los mismos horarios por la cuarentena del país. Se despidió a muchas TCP por falta de ingreso del empleador, no pudiendo acceder a los beneficios estatales debido a la informalidad. Los migrantes viven mayoritariamente en viviendas arrendadas, por lo que son más vulnerables frente al desempleo. Muchos colombianos acamparon delante del consulado de su país en Santiago, buscando ayuda por el aumento del desempleo. Finalmente, en julio del 2020, el Gobierno de Chile ayudó con un tercer avión de repatriación con destino a Bogotá, sin costo para las familias (Gissi et al, 2020; “Tercer vuelo de operativo...", 2020).

\section{Conclusión}

Muchas colombianas eligieron ir a Chile para trabajar porque hablan el mismo idioma, pensando que podrían ganar un mayor sueldo y pudiendo así ayudar a sus familiares en Colombia. Hasta antes del estallido social chileno, que empezó el 18 de octubre de 2019, y la llegada de la covid-19, el mercado laboral chileno favoreció la llegada de los extranjeros. La pandemia afectó gravemente al área del comercio, hotelería, además del trabajo de las trabajadoras de casa particular, donde la mayoría de las colombianas trabajan. Con este estudio se observó que ante una emergencia sanitaria o una crisis, como el estallido social, los migrantes son los más vulnerables, especialmente las mujeres que viajan sin familia. En Chile falta una regularización y un control más fuerte para luchar contra la informalidad del mercado laboral, además de la importancia de facilitar la contratación temporal. Debido a la reciente crisis social, al debilitamiento de la economía y finalmente a la llegada de la covid-19, que en especial genera incertidumbre en el mercado laboral, es más probable que el sueño chileno esté a punto de terminar para muchos migrantes, debido a la imposibilidad de establecerse en Chile.

\section{Referencias bibliográficas}

Alonso, Carlos - Sánchez, Dayana (2019). Informalidad de trabajadoras de casa particular supera el 50\% y vuelve a niveles previos a nueva ley. La Tercera. Asequible en: https://www.latercera.com/pulso/noticia/informalidad-de-trabajadoras-de-casa-particularsupera-el-50-y-vuelve-a-niveles-previos-a-nueva-ley/848774, fecha de consulta: 08-10-2020. Albertini, Marco - Mantovani, Debora y Gasperoni, Giancarlo (2019). Intergenerational relations among immigrants in Europe: the role of ethnic differences, migration and 
Amadea Bata-Balog - Gabriella Thomázy

acculturation. Journal of Ethnic and Migration Studies, 45(10). 1693-1706. DOI: 10.1080/1369183X.2018.1485202.

Arriagada, Irma - Moreno, Marcela (2011). La constitución de las cadenas globales de cuidado y las condiciones laborales de las trabajadoras peruanas en Chile. En C. Stefoni (ed.). Mujeres inmigrantes en Chile. ¿Mano de obra o trabajadoras con derecho? Santiago, Chile: Ediciones UAH. 149-192.

Baeza, Pablo (2019). Incorporación de inmigrantes sudamericanos en Santiago de Chile: Redes migratorias y movilidad ocupacional. Migraciones Internacionales, 10. 1-27. DOI: 10.33679/rmi.v1i1.2145.

Banco de la República (2019). Remesas de trabajadores. banrep.gov.co. Asequible en: https://www.banrep.gov.co/es/estadisticas/remesas, fecha de consulta: 08-11-2020.

Bata-Balog, Amadea - Thomázy, Gabriella (2020). Trends and Determinants of Colombian Migration to Chile. International Academic Institute. 67-81. Asequible en: https://ia-institute.com/iai-academic-conference-proceedings-barcelona-2020/, fecha de consulta: 05-10-2020.

Contreras, Pablo (2018). En dónde trabajan los extranjeros que han llegado a Chile. Publimetro. Asequible en: https://www.publimetro.cl/cl/noticias/2018/05/28/dondetrabajan-los-extranjeros-llegado-chile.html, fecha de consulta: 05-10-2020.

Corruption perceptions index 2019. Transparency International. Asequible en https://www.transparency.org/en/cpi/2019/results/, fecha de consulta: 10-10-2020.

Datos migratorios en América del sur (2020). Migraciondataporta.org. Asequible en https:/ / migrationdataportal.org/es/regional-data-overview/datos-migratorios-enamerica-del-sur, fecha de consulta: 09-11-2020.

DEM (2019). Estadísticas Migratorias. Extranjeria.gob.cl. Asequible en: https://www.extranjeria.gob.cl/estadisticas-migratorias, fecha de consulta: 01-05-2020.

DNP (2017). DNP inicia caracterización de los colombianos residentes en el exterior. Departamento Nacional de Planeación dnp.gov.co. Asequible en:

https://www.dnp.gov.co/Paginas/DNP-inicia-caracterizaci $\%$ C3\%B3n-de-los-

colombianos-residentes-en-el-exterior-.aspx, fecha de consulta: 13-10-2020.

El sueño de modelar terminó en explotación sexual. La Patria. 11 de noviembre de 2019. Asequible en: https://www.lapatria.com/sucesos/el-sueno-de-modelar-terminoen-explotacion-sexual-447805, fecha de consulta: 25-10-2020.

Fernández, Juan - Díaz, Vivian - Aguirre, Tatiana - Cortínez, Valentina (2020). Mujeres colombianas en Chile: discursos y experiencia migratoria desde la interseccionalidad. Revista Colombiana de Sociología, 43(1), 17-36. DOI: 10.15446/rcs.v43n1.79075.

Gissi, Nicolás - Pinto, Carolina - Rodríguez, Francisca (2019). Inmigración reciente de colombianos y colombianas en Chile. Sociedades plurales, imaginarios sociales y estereotipos. Estudios Atacameños, 62. 127-141. DOI: 10.22199/issn.0718-1043-2019-0011. 
Colombianas en Chile: tendencias y determinantes de la migración femenina

Gissi, Nicolás - Ghio, Gonzalo (2017). Estudio del proceso de integración y exclusión de los inmigrantes colombianos en la Región Metropolitana de Chile. Extranjeria.gob.cl. Asequible en: https://www.extranjeria.gob.cl/media/2019/04/DEMInvestiga2Estudio delProcesodeIntegracionyExclusiondelosInmigrantesColombianosenlaRegionMetropoli tanaChile.pdf, fecha de consulta: 08-10-2020.

Gissi, Nicolás - Galaz, Caterine y Facuse, Marisol (2020). Migración, crisis sanitaria y desigualdades sociales. Desafíos de la pandemia a la política migratoria en Chile. Uchile.cl. Asequible en: http://www.facso.uchile.cl/noticias/163455/migracion-crisis-sanitaria-ydesigualdades-sociales, fecha de consulta: 12-10-2020.

HDI (2019). hdr.undp.or. Asequible en http://hdr.undp.org/en/countries, fecha de consulta: 08-10-2020.

Hochschild, Arlie (2001). Las cadenas mundiales de afecto y asistencia y la plusvalía emocional. En: Giddens, A. y Hutton, W. (eds.) En el limite. La vida en el capitalismo global. Barcelona, España: Tusquets Ediciones. 187-208.

ILO (2018). Global Estimates on International Migrant Workers. Geneva: ILO. Asequible en: https://www.ilo.org/wcmsp5/groups/public/---dgreports/---dcomm/--publ/documents/publication/wcms_652001.pdf, fecha de consulta: 22-05-2020.

ILO (2017). Mujeres migrantes en Chile: oportunidades y riesgos de cruzar fronteras para trabajar. Ilo.org. Asequible en: https://www.ilo.org/wcmsp5/groups/public/--americas/---ro-lima/---sro-santiago/documents/publication/wcms_560975.pdf, fecha de consulta: 25-05-2020.

INE (2018). Características de la inmigración internacional en Chile - Censo 2017. Ine.cl. Asequible en: http://www.censo2017.cl/descargas/inmigracion/181123documento-migracion.pdf, fecha de consulta: 08-10-2020.

INE (2019). Estadísticas sociales. Ine.cl. Asequible en: https://www.ine.cl/ estadisticas/sociales/demografia-y-vitales/demografia-y-migracion, fecha de consulta: 14-10-2020.

IOM (2017). GMDAC Data Briefing - Measuring Global Migration Potential, 20102015. Asequible en: https://gmdac.iom.int/gmdac-data-briefing-measuring-globalmigration-potential-2010-2015, fecha de consulta: 08-03-2020.

Leal, Adriana (2020). Los profesionales ganan 71\% más que las personas que cuentan con básica primaria. La República. Asequible en: https://www.larepublica.co/ economia/los-profesionales-ganan-71-mas-que-personas-con-personas-con-basicaprimaria-2960985, fecha de consulta: 25-10-2020.

Ley N 18.620 Código del Trabajo, Chile, 16 de enero de 2003.

Ley No 20.786 Código del Trabajo, Chile, 27 de octubre de 2014.

López, Joaquín (2020). Los recursos que entraron al país alcanzaron un récord de US\$6.773 millones. La República. Asequible en: https://www.larepublica.co/economia 
/ las-remesas-que-llegaron-a-colombia-en-2019-significaron-us185-millones-diarios2957785, fecha de consulta: 08-10-2020.

O’Neil, Tam - Fleury, Anjali - Foresti, Marta (2016). Women on the move Migration, gender equality and the 2030 Agenda for Sustainable Development. Odi.org. Asequible en: https://www.odi.org/sites/odi.org.uk/files/resource-documents/10731.pdf, fecha de consulta: 13-10-2020.

ONU (2019). Statistics. Population Division, International Migration. Asequible en: https://www.un.org/en/development/desa/population/migration/data/estimates2/es timates19.asp, fecha de consulta: 30-03-2020.

Oso, Laura - Martínez, Raquel (2008). Domésticas y cuidadoras: mujeres inmigrantes latinoamericanas y mercado de trabajo en España. L'Ordinaire des Amériques, 208-209. 143-161. DOI: 10.4000/orda.3295.

Pavez, Jorge (2016). Afecciones afrocolombianas: Transnacionalización y racialización del mercado del sexo en las ciudades mineras del norte de Chile. Latin American Research Review, 51(2), 24-45. DOI: 10.1353/lar.2016.0021.

Polémica ley migratoria avanza en el Parlamento chileno. Efe, 1 de octubre de 2020. Asequible en: https://www.efe.com/efe/america/politica/polemica-ley-migratoriaavanza-en-el-parlamento-chileno/20000035-4356667, fecha de consulta: 12-10-2020.

Prado, Daniel (2018). "Antofalombia": cómo viven los colombianos que buscan el "sueño chileno" en Antofagasta. BBC Mundo. Asequible en: https://www.bbc.com /mundo/noticias-america-latina-42609403, fecha de consulta: 08-10-2020.

Thomázy Gabriella (2020). Tendencias actuales y nuevos desafíos de los migrantes en Chile. Acta Hispanica, (II), 409-421. DOI: 10.14232/actahisp.2020.0.409-421.

Tercer vuelo de operativo conjunto de repatriación lleva a colombianos a su país. Minrel, 23 de julio de 2020. Asequible en: https://minrel.gob.cl/tercer-vuelo-deoperativo-conjunto-de-repatriacion-lleva-a-colombianos-a/minrel/2020-07-

23/153023.html, fecha de consulta: 08-10-2020.

Insight Crime (2019). Balance de los homicidios en 2019. Insight Crime. Asequible en: https://es.insightcrime.org/noticias/analisis/balance-homicidios-2019/, fecha de consulta: 08-10-2020.

World Bank (2019a). Chile. Worldbank. Asequible en: https://data.worldbank.org /country/chile, fecha de consulta: 08-10-2020.

World Bank (2019b). Colombia. Worldbank. Asequible en: https://data.worldbank.org /country/colombia, fecha de consulta: 08-10-2020.

$48,5 \%$ de las mujeres participa en el mercado laboral. Ine.cl, 5 de marzo de 2018. Asequible en: https://www.ine.cl/prensa/2019/09/16/solo-un-48-5-de-las-mujeres-participa-en-elmercado-laboral-chileno-durante-2017-menor-al-71-2-de-participaci $\%$ C3\%B3n-de-loshombres, fecha de consulta: 17-10-2020. 